\title{
An avirulent Brachyspira hyodysenteriae strain elicits intestinal $\lg A$ and slows down spread of swine dysentery
}

\author{
Maxime Mahu (1), Filip Boyen, Stefano Canessa, Jackeline Zavala Marchan, Freddy Haesebrouck, An Martel \\ and Frank Pasmans*
}

\begin{abstract}
Swine dysentery caused by Brachyspira hyodysenteriae, results in substantial economic losses in swine producing countries worldwide. Although a number of different vaccine approaches have been explored with regard to this disease, they show limitations and none of them have reached the market. We here determine the vaccine potential of a weakly haemolytic $B$. hyodysenteriae strain. The virulence of this strain was assessed in experimental infection trials and its protection against swine dysentery was quantified in a vaccination-challenge experiment using a seeder infection model. Systemic IgG production and local IgA production were monitored in serum and faeces respectively. Across all trials, pigs that were colonized by virulent, strongly haemolytic B. hyodysenteriae strains consistently developed swine dysentery, in contrast to none of the pigs colonized by the weakly haemolytic $B$. hyodysenteriae vaccine strain. In the seeder vaccination trial nearly all immunised animals developed swine dysentery on subsequent challenge with a virulent strain, but the speed of spread of swine dysentery and faecal score were significantly reduced in animals immunised with the weakly haemolytic strain compared to sham-immunised animals. The IgA response of immunised animals upon challenge with a virulent $B$. hyodysenteriae strain significantly correlated to a later onset of disease. The correlation between local IgA production and protection induced by a weakly haemolytic $B$. hyodysenteriae strain provides leads for future vaccine development against swine dysentery.
\end{abstract}

\section{Introduction}

Swine dysentery (SD) caused by Brachyspira hyodysenteriae (B. hyodysenteriae), results in substantial economic losses in swine producing countries worldwide. Major costs associated with SD comprise medical treatment, retarded growth and increased feed conversion [1]. Treatment with antimicrobial compounds is hampered due to increasing resistance against lincosamides, pleuromutilins and macrolides which are the most widely used compounds against SD [2-4]. Besides instigating therapeutic failure, there is growing public concern against the use of antibiotics in animal production in general because it may favour spread of antimicrobial resistance in different

\footnotetext{
*Correspondence: frank.pasmans@ugent.be

Department of Pathology, Bacteriology and Avian Diseases,

Faculty of Veterinary Medicine, Ghent University, Salisburylaan 133,

9820 Merelbeke, Belgium
}

bacterial species, including zoonotic agents $[5,6]$. The impact of SD on swine health and production, increasing therapeutic failure of antimicrobial treatment and the need for a reduction of the use of antimicrobial compounds urge for alternative control measures against SD.

The immunological response in pigs that recovered from SD has been shown to protect against subsequent challenge with B. hyodysenteriae [7]. Therefore, a number of different vaccination approaches have been explored with regard to SD. Several reports describe the use of whole cell bacterins [8-12] or protein digests of whole cell bacterins [13-15]. Some of these bacterins, administered intramuscularly or intravenously, induce partial protection, demonstrated by a lower proportion of animals developing clinical SD, or animals developing less severe disease signs of SD [8-10, 16]. In contrast, Olson et al. [12] described animals developing a more severe form of SD with an earlier onset after vaccination with 
an inactivated $B$. hyodysenteriae vaccine. A major downside of the use of inactivated whole cell bacterins is that they usually only evoke protection against infection with a homologous serotype of $B$. hyodysenteriae [1].

Vaccination with recombinant proteins has been reported to induce variable levels of protection, depending on the selected protein. The use of a recombinant flaB1 flagellar protein could not reduce the number of pigs developing SD after challenge with a virulent $B$. hyodysenteriae strain [17]. A preparation of $\mathrm{BmpB}$, an outer membrane lipoprotein, resulted in a $50 \%$ reduction in clinical SD [18]. Song et al. [19] described a reverse vaccinology approach to select proteins for use in a subunit vaccine. They also reported a reduction in number of animals developing clinical SD, albeit not significant.

DNA vaccines based on $f t n A$, encoding a putative ferritin protein, or $S m p B$, encoding a protein with unknown function, failed to protect mice against challenge with a virulent $B$. hyodysenteriae strain $[20,21]$. The use of DNA vaccines for SD has not been investigated in pigs. A tlyA mutant strain of $B$. hyodysenteriae has been examined for its use as a live attenuated vaccine. A $50 \%$ reduction in the number of animals developing clinical SD upon challenge with a virulent $B$. hyodysenteriae strain was demonstrated. However, there was no reduction in the number of animals that was colonised by the challenge strain [22].

Despite all these efforts, an efficient vaccine against $B$. hyodysenteriae is currently not available. Recently, we isolated a weakly haemolytic $B$. hyodysenteriae strain which appeared to be less virulent than strongly haemolytic $B$. hyodysenteriae strains [23]. In this study we explore this strain's vaccination potential by verifying its virulence in pigs and determining the extent of protection it provides against SD in an experimental infection trial.

\section{Materials and methods}

The animal experiments were approved by the Ethical Committee of the Faculty of Veterinary Medicine, Ghent University, Belgium (EC 2012/01, EC 2013/147,
EC2014/130, EC2015/22, EC2015/134) and complied with all ethical and husbandry regulations.

\section{Brachyspira hyodysenteriae strains and growth conditions}

Three B. hyodysenteriae field strains and the strongly haemolytic reference strain B204 (ATCC32121) were used in the experimental infection trials: weakly haemolytic strain D28 and strongly haemolytic strain 8dII are two field strains which have been described previously [23]. Strongly haemolytic strain 49 was isolated in this study from seeder animals that were purchased from a commercial source suffering an acute outbreak of SD. Strains and their strength of haemolysis are given in Table 1. Strength of haemolysis was determined as visible haemolysis of growth on blood supplemented culture plates and by in vitro quantification as described in a previous study [23].

For the virulence trials, strains were obtained from frozen stocks, thawed and grown on Tryptic Soy Agar (BD, Heidelberg, Germany), supplemented with 5\% sheep blood (IMP, Brussels, Belgium) and 1\% yeast extract (Oxoid, Aalst, Belgium) [24]. Strains were subcultured twice and suspensions were prepared by harvesting a 4-day-old culture plate with a sterile cotton swab and stirring the cotton swab in $50 \mathrm{~mL}$ of an anaerobic Brain Heart Infusion (BHI) broth, supplemented with $10 \%$ of Foetal Bovine Serum (FBS). The broth was incubated anaerobically during $40 \mathrm{~h}$ on a rocking platform at $37^{\circ} \mathrm{C}$. After incubation, cultures were microscopically examined for purity and each animal was administered $40 \mathrm{~mL}$ of $B$. hyodysenteriae culture which contained approximately $1 \times 10^{8}$ colony forming units per $\mathrm{mL}$. For the seeder vaccination trial, cultures were obtained in grossly the same way as for the virulence trials except that bacteria were grown in $\mathrm{BHI}$ broth with $10 \% \mathrm{FBS}$ for $30 \mathrm{~h}$, after which the anaerobe broth was centrifuged at $1500 \mathrm{~g}$ for $20 \mathrm{~min}$ and the pellet was suspended in a volume leading to a final concentration of approximately $1 \times 10^{9} \mathrm{~B}$. hyodysenteriae per $\mathrm{mL}$.

Table 1 Brachyspira hyodysenteriae strains, experimental set-up and results for faecal excretion and clinical signs of SD

\begin{tabular}{|c|c|c|c|c|c|}
\hline $\begin{array}{l}\text { Virulence } \\
\text { trial number }\end{array}$ & $\begin{array}{l}\text { B. hyodysenteriae } \\
\text { strain }\end{array}$ & $\begin{array}{l}\text { Strength } \\
\text { of haemolysis }\end{array}$ & $\begin{array}{l}\text { Model and inoculation } \\
\text { route }\end{array}$ & $\begin{array}{l}\text { Number of pigs } \\
\text { positive for faecal } \\
\text { excretion }\end{array}$ & $\begin{array}{l}\text { Number of pigs } \\
\text { with SD }\end{array}$ \\
\hline 1 & $8 d l l$ & Strong & Direct oral inoculation & $2 / 6$ & $2 / 6$ \\
\hline 2 & B204 & Strong & Direct gastric inoculation & $5 / 9$ & $5 / 9$ \\
\hline 3 & 49 & Strong & Seeder model & $5 / 14$ & $4 / 14$ \\
\hline 4 & $\mathrm{D} 28$ & Weak & Direct oral inoculation & $5 / 8$ & $0 / 8$ \\
\hline 5 & $\mathrm{D} 28$ & Weak & Direct gastric inoculation & $4 / 12$ & $0 / 12$ \\
\hline $\begin{array}{l}\text { Seeder vaccination } \\
\text { experiment }\end{array}$ & $\mathrm{D} 28$ & Weak & Direct oral inoculation & $25 / 30$ & $0 / 30$ \\
\hline
\end{tabular}

a Given as proportion of total number of inoculated pigs. 


\section{Virulence trials}

In order to determine the in vivo virulence of different $B$. hyodysenteriae strains, several experimental infection trials were conducted. The correlation between faecal shedding, as a proxy of intestinal colonization, and faecal score, as a measure for the development of SD, was determined independently for the four different B. hyodysenteriae strains in five experimental infection trials. Experiments were conducted separately in different time periods. In each experiment a single strain was used. Strain D28 was used in two independent experiments.

Experimental set-up The five experimental set-ups are presented in Table 1 . In all set-ups, experimental animals were purchased from commercial sources with no prior history of SD. On arrival, faeces were collected from all individual animals and examined for the presence of Salmonella sp. by microbial culture as previously described [25] and for the presence of B. hyodysenteriae by microbial culture and qPCR [26]. All animals were fed a commercial starter feed ad libitum.

Experimental procedures Inoculation was performed on three consecutive days and was preceded by a $12 \mathrm{~h}$ fast. Inoculation was performed orally or intragastrically as given in Table 1. For intragastric inoculation, animals were anaesthetized by intramuscular injection with a combination of xylazine at $4.4 \mathrm{mg} / \mathrm{kg}$ (Xyl-M $2 \%{ }^{\circledR}$, VMD, Arendonk, Belgium) and zolazepam/tiletamine at $2.2 \mathrm{mg} / \mathrm{kg}$ (Zoletil ${ }^{\circledR} 100$, Virbac, Carros, France). All intragastrically inoculated animals were pretreated $90 \mathrm{~min}$ before inoculation with $0.75 \mathrm{mg} / \mathrm{kg}$ ranitidine (Zantac $^{\mathrm{TM}}$, GlaxoSmithKline, Genval, Belgium) to reduce stomach acid production.

In experiment 3 instead of direct inoculation, the contact animals (receivers) were placed in the same unit with animals that were shedding $B$. hyodysenteriae and had been confirmed to have SD (seeders). These seeder animals were purchased from a commercial source suffering an acute outbreak of SD. Strain 49 was isolated from faecal samples of those pigs.

Follow-up In all trials, animals were observed daily for the presence of diarrhoea and other disease signs. Two to three times a week, faeces were scored and stool samples were collected. Faecal scores were determined as 0: normal, 1: softer but formed, 2: unformed semiwet, 3: runny, 4: runny with mucus and blood. Scores 2 and 3 were supplemented with 0.5 if blood or mucus were present. DNA was extracted from the stool samples using a Qiagen Stool Mini Kit (Qiagen, Hilden, Germany) and the extracted DNA was used to determine the quantity of B. hyodysenteriae DNA with qPCR [26]. Correlation between faecal excretion of the strain used for inoculation and faecal score was determined for each experiment.
At the end of the trial (3-5 weeks after inoculation) or $24 \mathrm{~h}$ (trial 2) after the first signs of swine dysentery, animals were euthanized. During necropsy, tissue samples of the apex of the colon were collected in $10 \%$ buffered formalin for histology during necropsy. Animals were anaesthetized with a combination of xylazine at $4.4 \mathrm{mg}$ / $\mathrm{kg}$ (Xyl-M $2 \%{ }^{\circledR}$, VMD, Arendonk, Belgium) and zolazepam/tiletamine at $2.2 \mathrm{mg} / \mathrm{kg}$ (Zoletil ${ }^{\circledR} 100$, Virbac, Carros, France). They were euthanized by administering an overdose of pentobarbital (Release ${ }^{\circledR}, 45 \mathrm{mg} / \mathrm{kg}$; Ecuphar, Oostkamp, Belgium) by intracardial injection. Fixed samples were paraffin embedded, sectioned at 5-8 $\mu \mathrm{m}$ and stained with hematoxylin and eosin or with Periodic Acid Schiff reagent (PAS).

\section{Vaccination trials}

In the seeder vaccination trial, non-virulent $B$. hyodysenteriae strain D28 was used as the immunising strain, and virulent $B$. hyodysenteriae strain B204 as the challenge strain. Sixty, 6-week-old male and female piglets were purchased from a commercial source with no previous history of SD. On arrival, animals were weighed and randomly assigned to six groups; three immunisation groups (10 animals each) and three non-immunisation groups (10 animals each). Individual faecal samples were taken to confirm absence of $B$. hyodysenteriae by microbial culture and qPCR. All animals were fed a commercial starter feed ad libitum.

After a 9-day acclimatization period, animals in the immunisation groups were orally inoculated as described for the virulence trials on three consecutive days (day -2 , day -1 , day 0 ) with $20 \mathrm{~mL}$ of a culture containing approximately $10^{9}$ colony forming units $(\mathrm{cfu}) / \mathrm{mL}$ of nonvirulent strain D28. Correspondingly, animals in the nonimmunisation groups were orally inoculated with $20 \mathrm{~mL}$ of BHI broth supplemented with 10\% FBS. All animals were pre-treated $90 \mathrm{~min}$ before inoculation with $0.75 \mathrm{mg} /$ kg ranitidine (Zantac ${ }^{\mathrm{TM}}$, GlaxoSmithKline, Genval, Belgium) to reduce stomach acid production.

Three weeks after (sham-)immunisation, five animals of each group were challenged with virulent $B$. hyodysenteriae strain $\mathrm{B} 204$ on three consecutive days by oral inoculation (day 19, day 20, day 21) as described above for the immunising strain. These challenged animals served as seeder animals for the remaining five animals (receivers) in each group.

Animals were observed daily for the presence of diarrhoea and other disease signs. During the period postimmunisation until challenge, faecal samples were collected three times a week from immunised animals. From these faecal samples DNA was extracted as described above to determine excretion of the immunising strain and faeces were scored as described for the 
virulence trials. These faecal samples were also used to determine the presence of faecal IgA against $B$. hyodysenteriae. Faecal samples from non-immunised animals were collected once during this period to confirm the absence of $B$. hyodysenteriae and faecal IgA against $B$. hyodysenteriae. After challenge, faecal samples were taken two times a week from all animals. These samples were scored and DNA was extracted to determine the excretion of the immunising and/or challenge $B$. hyodysenteriae strain. These faecal samples were used to determine the presence of faecal IgA against B. hyodysenteriae as well.

Animals were weighed at the start of the trial (before immunisation), after immunisation at day 17 and at necropsy. Average daily weight gain was calculated for each individual animal. Blood samples for determination of the presence of $B$. hyodysenteriae reactive serum IgG were taken before immunization (day -13$)$ and before challenge (day 17).

Animals were euthanized at day 50-52 (30-32 days post challenge) or sooner if apathy or depression was noted. Euthanasia was performed as described for the virulence trials.

\section{qPCR differentiating between the immunising and the challenge strain}

In order to specifically determine the quantity of B. hyodysenteriae DNA of the immunising strain and the challenge strain in faecal and intestinal samples, primers were designed to specifically anneal with DNA of either strain. Primers were based on the haemolysin III gene from both strains: D28 (GenBank KU215635) and B204 (GenBank JXND01000108) [23, 27]. Following primers were used for specific detection of the immunising strain D28: HlyVacFo 5'TGGTGAAATACTGCCAAAA3' and HlyVacRe 5'TGTTGTTATATCGTCCATAC3'. Following primers were used to specifically detect the challenge

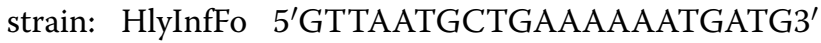
and HlyInfRe 5'AAGCTCTTGTATGGAATATAC3'. For both strains, following primer pair was used to generate an amplicon to be used as a standard: HlySTFo 5'CAAGTTCTATGATACCTAC3' and HlySTRe 5'GCCGCCTTTAACATAYTCTTT3'. The quantitative PCR was performed on a CFX96 ${ }^{\mathrm{TM}}$ RT-PCR System with a C1000 Thermal Cycler (Bio-Rad, Hercules CA, USA). Two $\mu \mathrm{L}$ of DNA was suspended in a $10 \mu \mathrm{L}$ reaction mixture consisting of SensiMix ${ }^{\mathrm{TM}}$ SYBR No-ROX (Bioline Reagents Ltd, UK), HPLC water and primers at $1.5 \mu \mathrm{M}$ for the challenge strain, and at $0.5 \mu \mathrm{M}$ for the immunising strain. The PCR program consisted of denaturation for $10 \mathrm{~min}$ at $95^{\circ} \mathrm{C}$, followed by 40 cycles of $95^{\circ} \mathrm{C}$ for $30 \mathrm{~s}$, and $60{ }^{\circ} \mathrm{C}$ for $30 \mathrm{~s}$. Standards and samples were run in duplicate. Reactions for both strains were performed separately, since both amplicons generated a melt temperature of $74.5{ }^{\circ} \mathrm{C}$ and could not be distinguished based on their melt temperatures. The Bio-Rad CFX Manager (version 1.6) software was used for calculation of threshold cycles $(C t)$-values and melting curve analysis of amplified DNA.

\section{Enzyme-linked immuno sorbent assay (ELISA) for specific detection of serum IgG and faecal IgA against $B$. hyodysenteriae}

For detection of antibodies against $B$. hyodysenteriae strains D28 or B204, in-house whole cell ELISAs were prepared for both strains as described previously for Salmonella enterica [28]. Each strain was grown in BHI with $10 \% \mathrm{FBS}$ for $48 \mathrm{~h}$ on a rocking platform at $37^{\circ} \mathrm{C}$. Cultures were inactivated by adding $0.18 \%(\mathrm{v} / \mathrm{v})$ formalin. The inactivated $B$. hyodysenteriae suspensions were washed with phosphate buffered saline (PBS) with $0.18 \%$ formalin $(\mathrm{v} / \mathrm{v})$ and finally resuspended in coating buffer $(1.08 \mathrm{~g}$ $\mathrm{Na}_{2} \mathrm{CO}_{3} \cdot 10 \mathrm{H}_{2} \mathrm{O}, 0.968 \mathrm{~g} \mathrm{NaHCO}_{3}, 0.25 \mathrm{~L}$ aqua ad injectabilia $100 \%$ w/v). F96 Nunc-immuno plates (Nunc International, Roskilde, Denmark) were coated with $140 \mu \mathrm{L}$ of inactivated B. hyodysenteriae in coating buffer, diluted to an optical density of 0.3 at $660 \mathrm{~nm}$. After a $24 \mathrm{~h}$-incubation period at $4{ }^{\circ} \mathrm{C}$, plates were washed three times with $100 \mu \mathrm{L}$ of wash buffer $\left(0.6 \mathrm{~g} \mathrm{NaH}_{2} \mathrm{PO}_{4} \cdot 2 \mathrm{H}_{2} \mathrm{O}, 5.6 \mathrm{~g}\right.$ $\mathrm{NaH}_{2} \mathrm{PO}_{4} \cdot 12 \mathrm{H}_{2} \mathrm{O}, 0.5 \mathrm{~mL}$ Tween 20, $12.5 \mathrm{~g} \mathrm{NaCl}$ ). Plates were kept at $4{ }^{\circ} \mathrm{C}$ until further use.

Wells were pre-incubated with $1 \%$ skim milk powder solution in distilled water for 15 min to block nonspecific binding. For detection of IgG in serum samples, $100 \mu \mathrm{L}$ of $1 / 200$ diluted sera were added to the wells and incubated for $30 \mathrm{~min}$ at room temperature. After incubation, wells were washed five times with wash buffer after which $100 \mu \mathrm{L}$ of a 1/20 000 dilution of a horseradish peroxidase conjugated anti-porcine IgG (Sigma-Aldrich, St. Louis, MO, USA) was added. After 30 min of incubation wells were washed five times with wash buffer and $100 \mu \mathrm{L}$ 3,3',5,5'-tetramethylbenzidine (Sigma-Aldrich, St. Louis, MO, USA) reagent was added. The enzymatic reaction was stopped after $10 \mathrm{~min}$ by adding $100 \mu \mathrm{L}$ of $1 \mathrm{~N} \mathrm{HCl}$. Optical densities were measured with a spectrophotometer (Multiskan MS, Thermofisher Scientific, Waltham, MA, USA) at $450 \mathrm{~nm}$.

For detection of IgA in faecal samples, extracts were prepared as described by Peeters et al. [29]. One gram of frozen faeces was weighed and placed on ice. Three $\mathrm{mL}$ of extraction buffer (PBS, 0.5\% Tween 20\%, 0.05\% $\mathrm{NaN}_{3}$ ) was added and the suspension was centrifuged at $4{ }^{\circ} \mathrm{C}$ for $20 \mathrm{~min}$ at $1500 \mathrm{~g}$. The supernatant was collected in a $2 \mathrm{~mL}$ Eppendorf tube (Eppendorf, Hamburg, Germany). Twenty $\mu \mathrm{L}$ of proteinase inhibitor (Sigma-Aldrich, St. Louis, MO, USA) was added before centrifugation for $10 \mathrm{~min}$ at $3000 \mathrm{~g}$ at $4{ }^{\circ} \mathrm{C}$. Supernatant was collected and stored at $-20{ }^{\circ} \mathrm{C}$ until further use. For detection of IgA 
reactive with $B$. hyodysenteriae in these faecal extracts, ELISA was carried out as for IgG detection in serum with following changes: faecal extracts were used undiluted and were incubated for $60 \mathrm{~min}$, the secondary antibody, goat anti-porcine IgA (Bio-rad, Kidlington, UK) was used in a $1 / 5000$ dilution.

\section{Statistical analysis}

Correlation between faecal excretion and faecal score was determined by Spearman's rank order correlation (r) and was performed with SPSS 22.0 software (SPSS Inc, Chicago, USA). In the seeder vaccination model, faecal scores were analysed using cumulative logit link regression. Interactions between time, type and treatment were included, as well as random effects at the individual level, to control for pseudo-replication in the individual time series, and at the pen effect to account for clustering. The analysis was performed using package "ordinal" in R. Analysis was repeated for faecal excretion, this time using a linear mixed model (package "lme4" in R), with the same predictors as above.

The effect of treatment on average daily weight gain was analysed using linear regression. The effect of faecal IgA response on the time of onset of SD was analysed in two steps. First, measurements for individual animals, collected at regular intervals $(3,6,10,13$, and 17 days post challenge), were analysed using a survival model, estimating whether the probability of an individual developing SD was delayed by a stronger faecal IgA response. The use of the survival model enabled us to account for censoring (some individuals had not developed disease by the time the experiment was terminated). To capture several possibilities, the analysis of the survival model was repeated using as response variable alternatively the maximum IgA value, the arithmetic mean and the geometric mean across all days before the first notice of disease signs for a given individual. The geometric mean was used to better reflect the possible dependency between successive measures of IgA in the same individual. All three survival models included a fixed effect for individual type (seeder/receiver) and a random effect at the pen level to account for clustering. All analyses of faecal IgA responses were carried out using the "survival" package in R. For all analyses, the statistical significance level was set at $\alpha=0.05$.

\section{Results}

\section{The low haemolytic B. hyodysenteriae strain D28 is} avirulent in pigs

In all virulence trials, faeces of all animals were negative for $B$. hyodysenteriae on arrival, although some animals of each trial tested positive for B. innocens. All animals tested negative for Salmonella. Across all infection trials, out of 12 pigs that were colonized by strongly haemolytic B. hyodysenteriae strains, 11 consistently developed SD, in contrast to none of the 34 pigs colonized by the weakly haemolytic strain D28 (Table 1). One receiver animal in experiment 3 shed B. hyodysenteriae in its faeces on two occasions at the end of the trial. At the same time the animal had a faecal score of 2 . It is possible that this animal would have developed SD in the following days. However, since the trial ended simultaneously for all animals in that trial, the animal was euthanized and the development of SD could not be confirmed.

In the seeder vaccination trial, during the period between immunisation with strain D28 and the challenge with the virulent strain, no clinical signs of dysentery were noticed in any animal. On all sampling occasions, maximum faecal score was 1 . Score 1 was noted in immunised as well as in non-immunised animals.

Figure 1 shows the correlation between faecal excretion and faecal scores for weakly haemolytic strain D28 (Figures $1 \mathrm{~A}-\mathrm{C}$ ) and for strongly haemolytic strains $8 \mathrm{dII}$ (Figure 1D), 49 (Figure 1E) and B204 (Figure 1F). The correlation between faecal excretion and faecal scores was significant $(p<0.01)$ for the strongly haemolytic strains and the correlation coefficients $(r)$ were 0.67 (strain 8dII), 0.40 (strain 49) and 0.64 (strain B204) respectively. For weakly haemolytic strain D28 there was no correlation between faecal excretion and faecal score in any of the experiments; experiment $4 \mathrm{r}=0.049, p=0.684$, experiment $5 \mathrm{r}=-0.064, p=0.536$, seeder vaccination experiment $\mathrm{r}=-0.007, p=0.919$.

All animals, inoculated with one of the strongly haemolytic $B$. hyodysenteriae strains, that showed clinical signs of SD had various lesions in large parts of the colon. Contents of the colon were liquid and macroscopic lesions consisted of serosal hyperaemia, fibrinous colitis, enlarged mesenteric lymph nodes and the presence of excessive mucus at the colonic mucosa. Histologically, elongation of the colonic crypts and presence of a large amount of mucoid material in the lumen of the infected animals were remarkable. The lamina propria mucosae was infiltrated by lymphocytes, plasma cells, and neutrophils.

Of the animals that shed weakly haemolytic B. hyodysenteriae strain D28, some showed slight hyperaemia of the colonic mucosa but no other apparent macroscopic lesions were observed. Histologically, no elongation of the colonic crypts was observed and no infiltration of inflammatory cells could be observed in the lamina propria.

PAS staining showed elongation of the colonic crypts and a high number of Goblet cells in animals colonized with a strongly haemolytic strain (Figure 2B; animal colonized with strongly haemolytic strain $8 \mathrm{dII}$ as an 


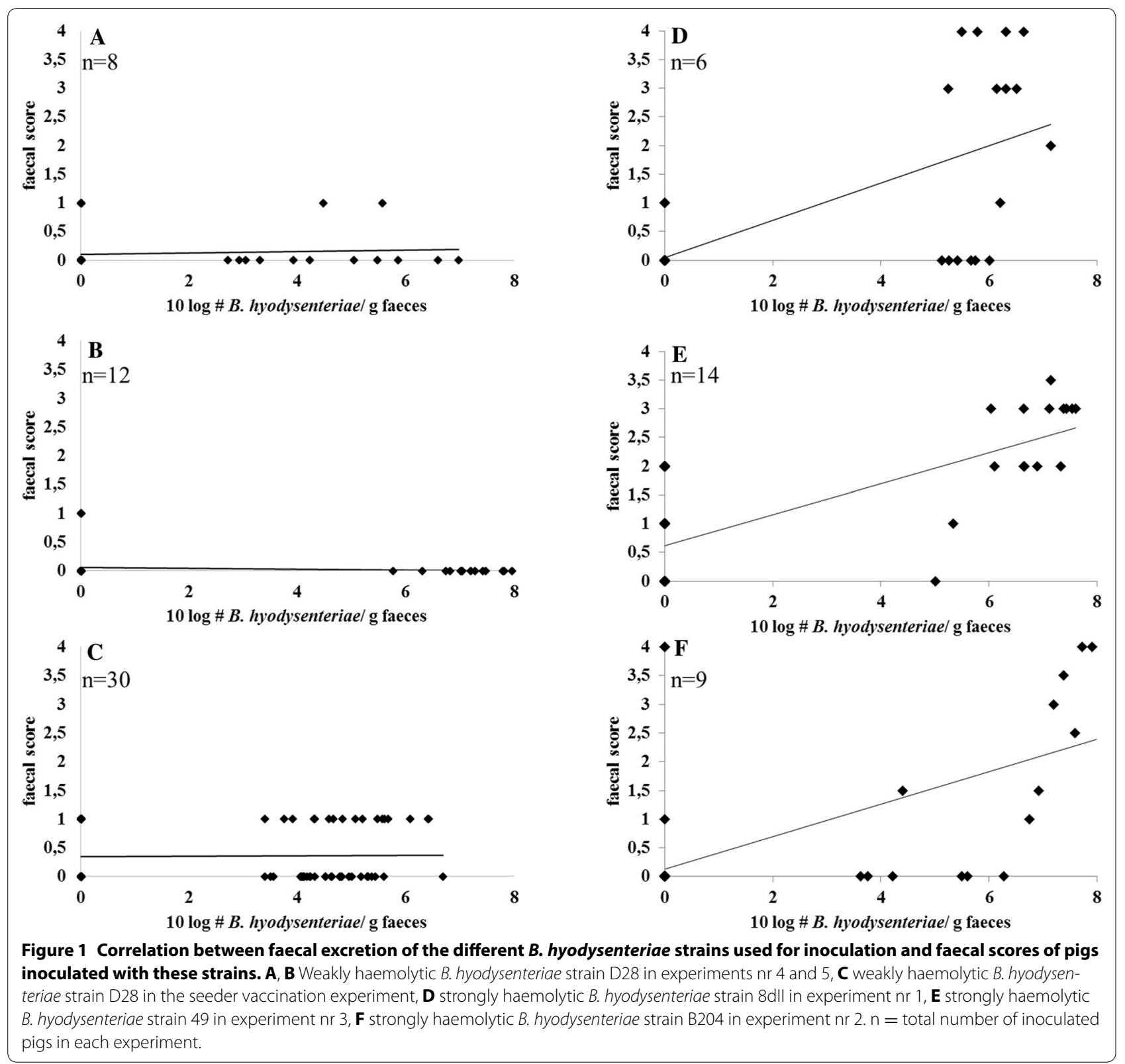

example), but not in those colonized with the weakly haemolytic strain D28 (Figure 2C) or in negative control animals (Figure 2A).

\section{Vaccination with the avirulent strain delays the spread of SD}

In the seeder vaccination trial, of the 30 animals inoculated with immunising strain D28, the majority shed the strain in their faeces for less than 1 week (12 animals) or less than 2 weeks (10 animals). Three animals shed the strain for more than 2 weeks and for five animals, strain D28 could not be detected with qPCR throughout the experiment. Most animals (18 out of 25) started shedding strain D28 in their faeces within the first week after inoculation, six animals in the second week, and one animal at 17 days post inoculation. During the period post vaccination until challenge vaccinated animals had a significantly lower average daily weight gain compared to sham-vaccinated animals (linear regression coefficient for treatment: $\beta=-0.52 \pm 0.2 \mathrm{SE}, p=0.010$ ).

The total number of animals that developed SD after challenge with the virulent $B$. hyodysenteriae strain is given in Figure 3. At the end of the trial 28/30 animals had developed SD in the immunised group as well as 

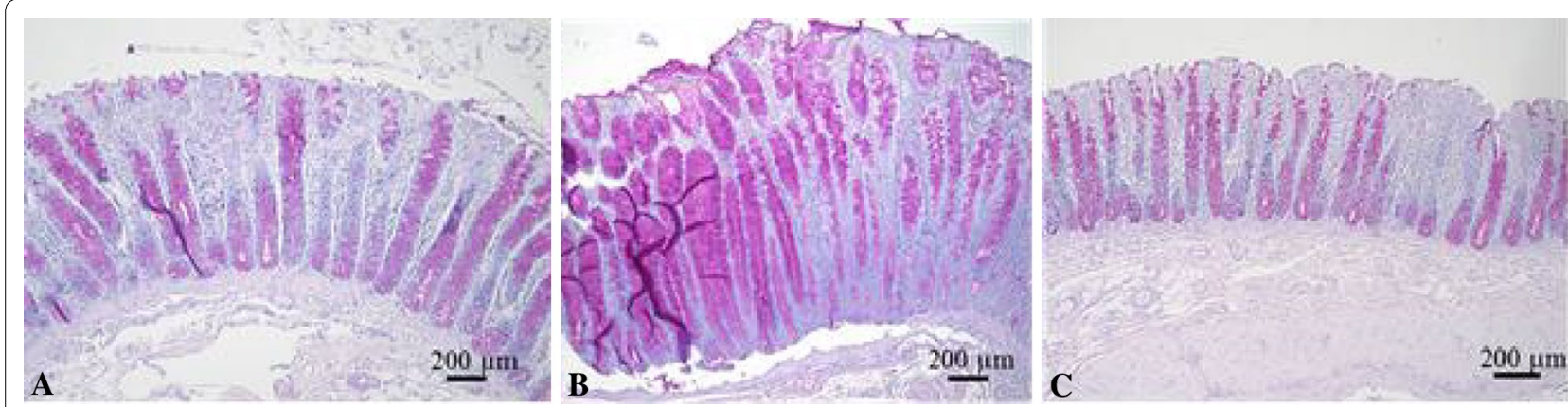

Figure 2 PAS staining of formalin fixed colonic tissue samples of pigs infected with different $B$. hyodysenteriae strains. Colonic mucosa from $\mathbf{A}$ sham inoculated animal, B animal infected by strongly haemolytic B. hyodysenteriae strain 8dll (33 days post inoculation), C animal infected by weakly haemolytic B. hyodysenteriae strain D28 (32 days post inoculation).

in the non-immunised group. The onset of SD, defined as the first day on which a faecal score of 2.5 or more was reached and mucus and/or blood were present, was postponed in immunised animals compared to non-immunised animals. There was a significant interaction between immunisation and number of days from challenge until onset of SD (regression estimate $\beta=0.065 \pm 0.022 \mathrm{SE}, p=0.004)$. Seeder animals in the immunisation groups developed SD on average after 11.1 (10-11.8) days while seeder animals in the non-immunisation groups developed SD on average after 9.2 days (7.6-12). Receiver animals developed SD on average after
21.2 days (18.8-22.4) in the immunised groups, and after 17.3 days (15.6-20.25) in the non-immunised groups.

The average cumulative faecal score of pigs after challenge with virulent $B$. hyodysenteriae strain B204 is given in Figure 4 . The main effects in the regression showed that the probability of having a higher faecal score decreased with immunisation (regression coefficient: $\beta=-1.398 \pm 0.384 \mathrm{SE}, p=0.0002$ ), indicating that immunisation significantly reduced the probability of having a higher faecal score.

We did not find a significant correlation between immunisation and the faecal excretion of the challenge

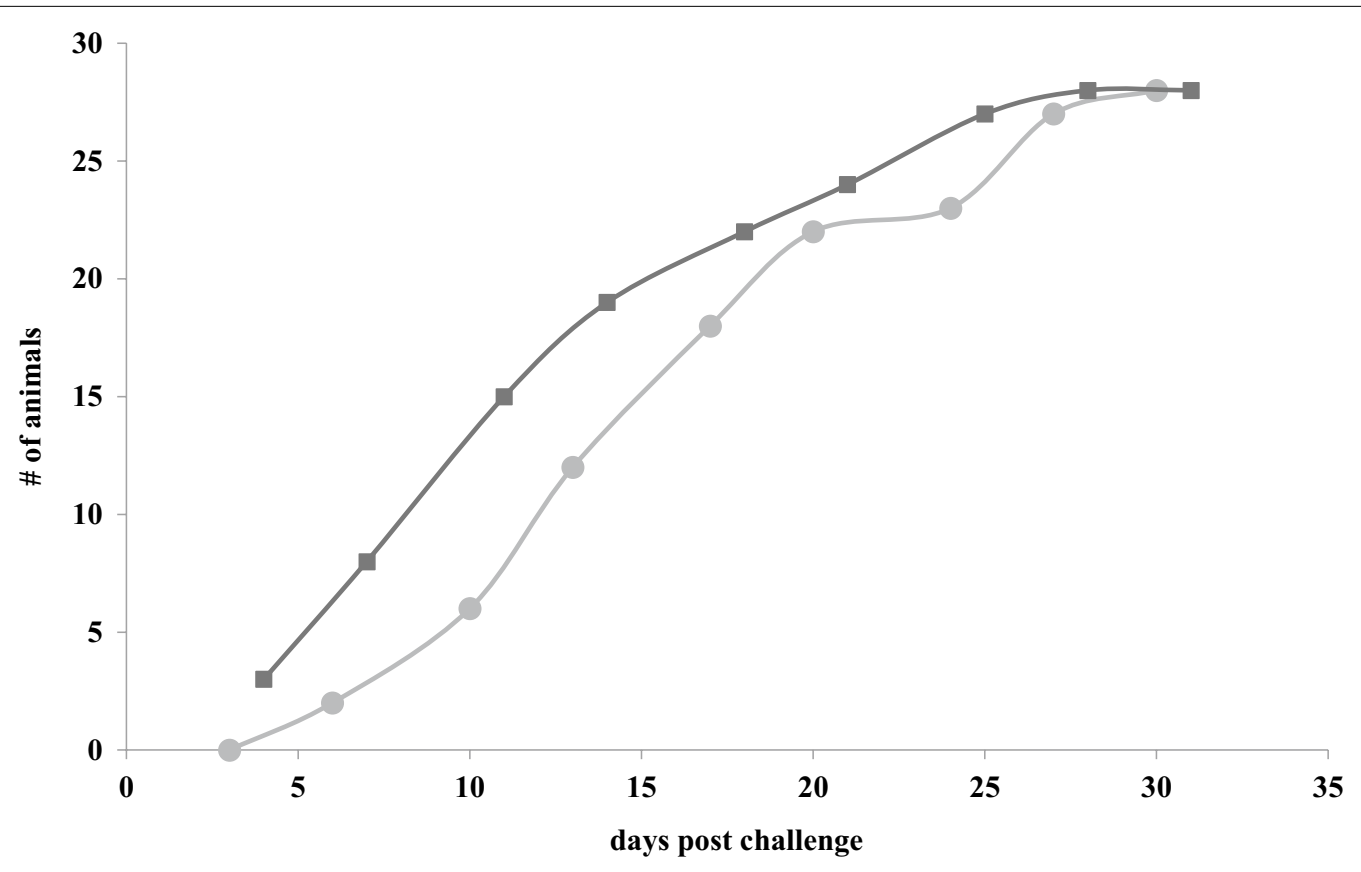

Figure 3 Cumulative number of pigs that developed SD after challenge with virulent B. hyodysenteriae strain B204, preceded either (circles) or not (squares) by exposure to non-virulent B. hyodysenteriae strain D28. 


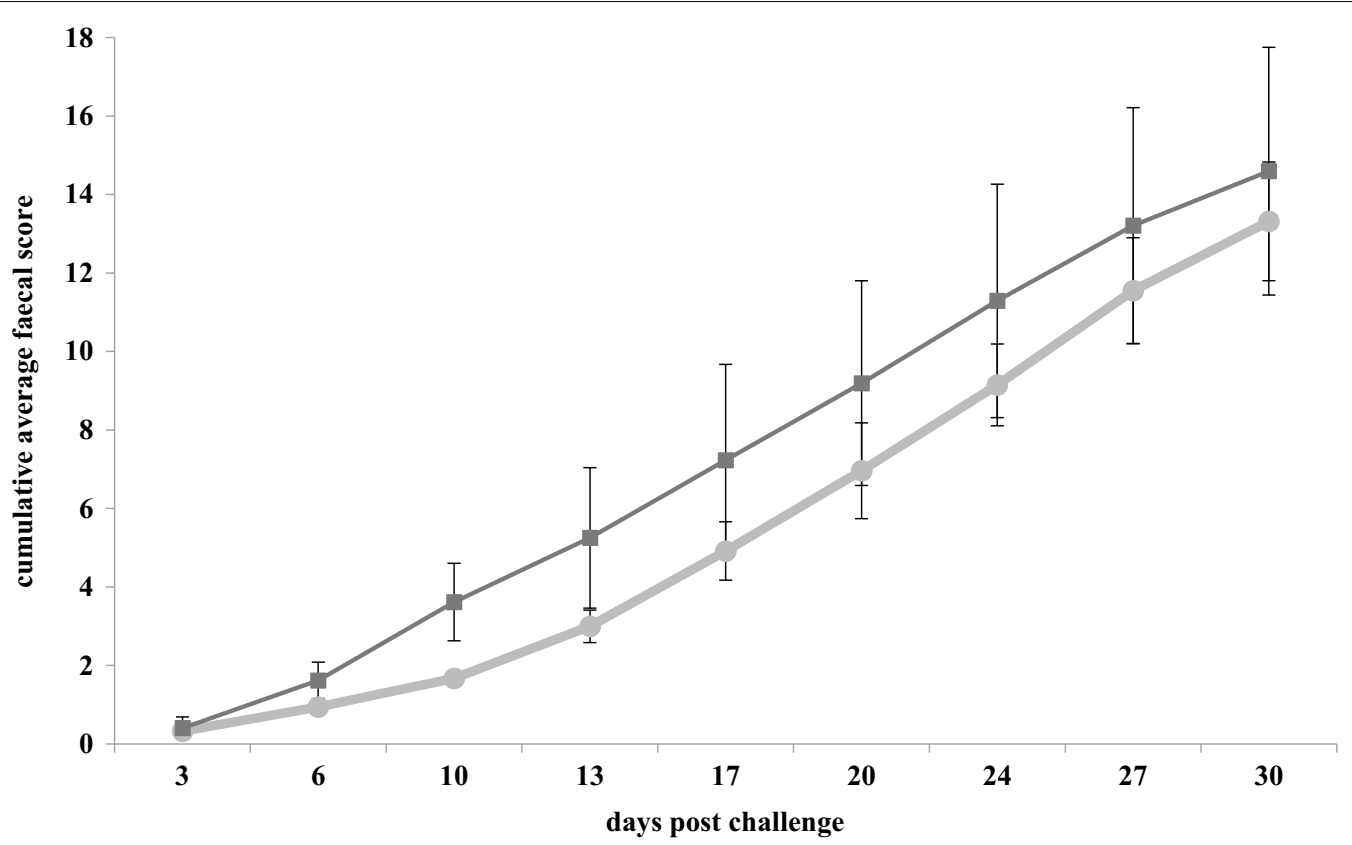

Figure 4 Average cumulative faecal score of pigs after challenge with virulent B. hyodysenteriae strain B204, preceded either (circles) or not (squares) by exposure to non-virulent $B$. hyodysenteriae strain D28.

strain (all $p>0.3$ ). Faecal excretion of the challenge strain was correlated with faecal scores, and equally strong for vaccinated and non-vaccinated animals (vaccinated animals $\mathrm{r}=0.79, p<0.001$, non-vaccinated animals $r=0.74, p<0.001$ ). In all weight gain analyses, no significant differences were found between seeders and receiver individuals. Although a trend could be observed for immunised animals, most noticeable for receiver animals, to have a higher weight gain in the post challenge period compared to non-immunised animals, this difference was not significant $(p=0.17)$. Plots of average daily weight gain are given in Figure 5.

The avirulent strain does not induce a fast lgG response Serum IgG ELISA responses against $B$. hyodysenteriae are given in Table 2. There was no significant increase
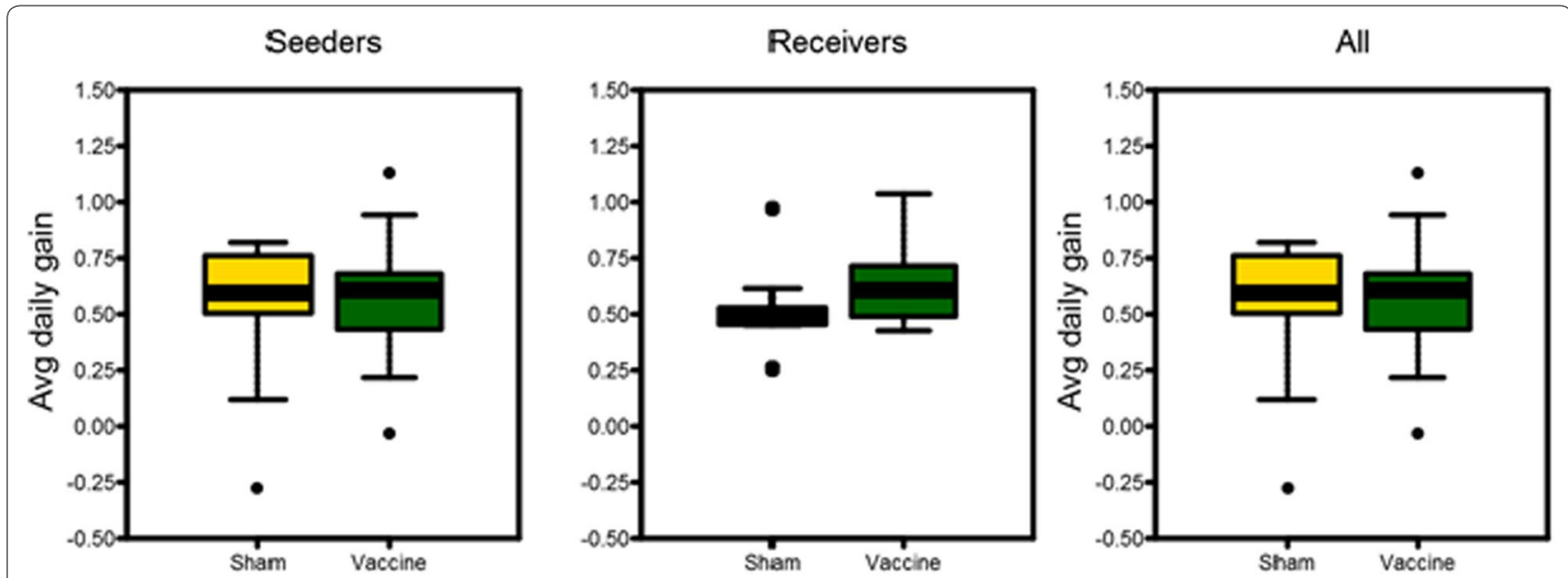

Figure 5 Plots of average daily weight gain (period: post-vaccination to necropsy), of pigs after challenge with virulent $B$. hyodysenteriae strain B204, preceded either (green) or not (yellow) by exposure to non-virulent B. hyodysenteriae strain D28, grouped by individual type (seeder, receiver and both) and by vaccination treatment. Boxes indicate 25 and $75 \%$ quantiles: bars indicate 2.5 and $97.5 \%$ quantiles. Average daily weight gain given in $\mathrm{kg}$. 
Table 2 Production of serum IgG against B. hyodysenteriae before and after vaccination with the avirulent strain D28

\begin{tabular}{llllll}
\hline Group & \multicolumn{2}{l}{ Whole cell ELISA, D28 coated } & & \multicolumn{2}{l}{ Whole cell ELISA, B204 coated } \\
\cline { 2 - 3 } & Pre vaccination (day - 13) & Post vaccination (day 17) & & Pre vaccination (day - 13) & Post vaccination (day 17) \\
\hline Vaccinated animals & $0.27 \pm 0.17$ & $0.31 \pm 0.13$ & $0.25 \pm 0.16$ & $0.23 \pm 0.06$ \\
Non-vaccinated animals & $0.19 \pm 0.14$ & $0.23 \pm 0.17$ & $0.21 \pm 0.14$ & $0.21 \pm 0.09$ \\
\hline
\end{tabular}

The reaction of $\lg G$ with both the vaccination strain (D28) and the challenge strain (B204) are shown and presented as $\mathrm{OD}_{450}$ values with standard deviation.

of IgG post-vaccination either for vaccinated or nonvaccinated animals, regardless of which strain was used to coat the whole cell ELISA. There were no significant differences between vaccinated and non-vaccinated animals, before or after vaccination.

\section{The avirulent strain induces a local yet variable $\lg A$ response}

During the period post-vaccination and before challenge with the virulent $B$. hyodysenteriae strain, faecal IgA increased in 11 of 30 vaccinated animals. The faecal IgA response was measured 7 times during this period and there were large individual differences. The faecal IgA response was measured in non-vaccinated animals once at the end of this period. For none of those animals there was an increase in IgA. Faecal IgA responses are given in Table 3 .

Faecal IgA responses were also determined in the period after challenge on days $3,6,10,13$, and 17 post challenge. To assess the effect of IgA on the delay of development of clinical SD, maximum IgA response and geometric mean IgA response of individual animals were determined and correlated to the time of onset of SD for that specific animal. Since delay in onset of SD was to be determined, only IgA values before the actual onset of $\mathrm{SD}$ for each animal were retained. Furthermore, we only considered seeder animals, since for receiver animals the time of first exposure to B. hyodysenteriae is unknown.
Non-vaccinated animals showed low levels of faecal IgA until onset of SD. For vaccinated seeder animals there was greater variability between individuals. The maximum IgA response and the geometric mean IgA response of those vaccinated seeder animals were significantly correlated to a later onset of disease (regression coefficient for geometric mean IgA: $\beta=7.26 \pm 3.59 \mathrm{SE}, p=0.043$, for maximum IgA response: $\beta=7.59 \pm 2.58 \mathrm{SE}$, $p=0.003)$. The maximum IgA and geometric mean $\operatorname{Ig} \mathrm{A}$ response, correlated with the time of onset of SD are shown in Figures 6 and 7.

\section{Discussion}

In the pigs inoculated with the three strongly haemolytic strains, a significant correlation between faecal excretion of B. hyodysenteriae and faecal scores was observed. In the two virulence trials using the weakly haemolytic strain D28, however, none of the nine animals that shed the strain in their faeces developed disease signs or lesions associated with SD. Moreover a faecal score of more than one was never observed and there was no correlation between shedding of the weakly haemolytic B. hyodysenteriae strain and an elevated faecal score. This lack of correlation was independently confirmed in the seeder vaccination trial, in which none of the 25 pigs that shed strain D28 in their faeces developed disease signs of $\mathrm{SD}$, and no correlation between shedding of this strain and elevated faecal scores could be observed. All these

Table 3 Faecal IgA production before and after vaccination with the avirulent B. hyodysenteriae strain D28

\begin{tabular}{|c|c|c|c|c|}
\hline \multirow[t]{2}{*}{ Days post vaccination } & \multicolumn{2}{|c|}{ Whole cell ELISA, D28 coated } & \multicolumn{2}{|c|}{ Whole cell ELISA, B204 coated } \\
\hline & Vaccinated animals & Non-vaccinated animals & Vaccinated animals & Non-vaccinated animals \\
\hline Day -13 & $0.12 \pm 0.14$ & $0.12 \pm 0.28$ & $0.11 \pm 0.13$ & $0.14 \pm 0.41$ \\
\hline Day 3 & $0.08 \pm 0.04$ & NA & $0.07 \pm 0.03$ & NA \\
\hline Day 5 & $0.15 \pm 0.28$ & NA & $0.11 \pm 0.13$ & NA \\
\hline Day 7 & $0.14 \pm 0.15$ & NA & $0.09 \pm 0.06$ & NA \\
\hline Day 10 & $0.34 \pm 0.70$ & NA & $0.13 \pm 0.18$ & NA \\
\hline Day 12 & $0.39 \pm 0.55$ & NA & $0.12 \pm 0.09$ & NA \\
\hline Day 14 & $0.66 \pm 0.99$ & NA & $0.25 \pm 0.47$ & NA \\
\hline Day 17 & $0.62 \pm 0.96$ & $0.11 \pm 0.06$ & $0.18 \pm 0.21$ & $0.10 \pm 0.06$ \\
\hline
\end{tabular}

The reaction of IgA with both the vaccination strain (D28) and the challenge strain (B204) are shown and presented as $\mathrm{OD}_{450}$ values with standard deviation. NA: not applicable. 


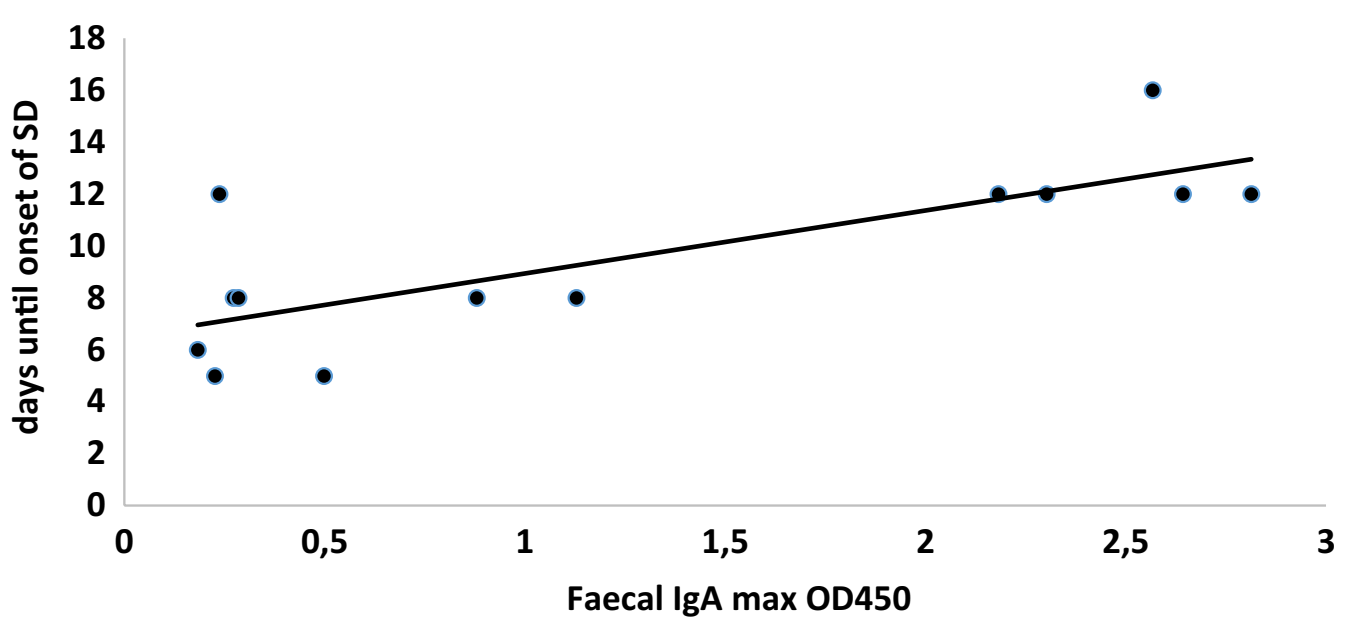

Figure 6 IgA max correlated with time of onset of SD (given in days post challenge with the virulent strain) for vaccinated seeder animals. $X$-axis presents the maximal OD450 values as measured in the ELISA.

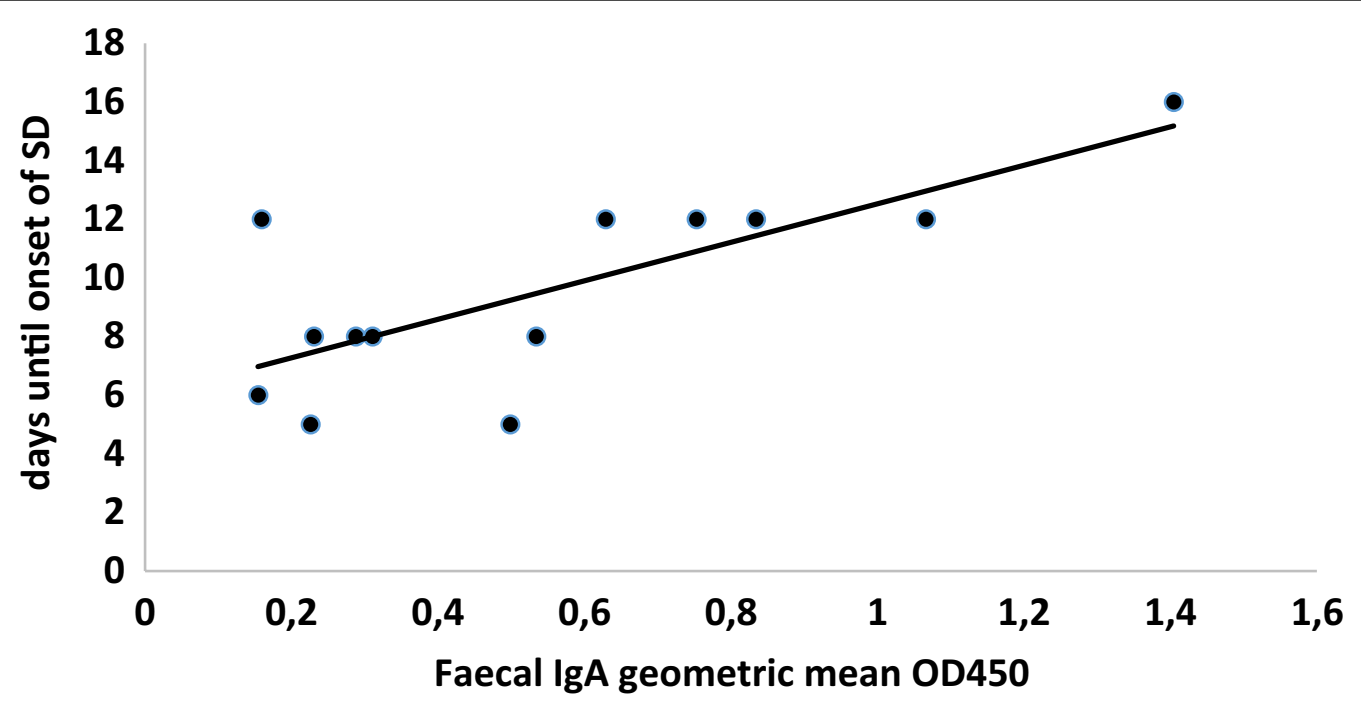

Figure 7 Geometric mean IgA correlated with time of onset of SD (given in days post challenge with the virulent strain) for vaccinated seeder animals. $X$-axis presents geometric mean of OD450 values as measured in the ELISA.

results strongly indicate that the weakly haemolytic $B$. hyodysenteriae strain D28 is avirulent in pigs.

La et al. [30] described reduced virulence in a weakly haemolytic $B$. hyodysenteriae strain obtained from a herd with no clinical signs of SD. In this herd, sows were present with substantial growth of $B$. hyodysenteriae strain JR11 in their colon, without any clinical sign of SD. Although true virulence potential has not yet been verified under experimental conditions, authors described strain JR11 as particularly innocuous [30]. Interestingly, strains D28 and JR11 share almost identical differences in their haemolysin III and haemolysin activation protein, as given for strain D28 earlier [23, 30]. Compared to the whole genome sequence of reference strain WA1 (Accession Number NC_012225), both weakly haemolytic strains JR11 and D28 show five identical amino acid substitutions (positions $81,113,164,227,265$ ) in the haemolysin activation protein BHWA1_RS02885 and eight identical amino acid substitutions in the haemolysin III protein BHWA1_RS02195 (positions 47, 49, 56, 79, 82, 111, 114, 133). For strain JR11 two additional amino acid substitutions were identified in the haemolysin III protein at positions 30 and 213. It should be noted that unintentionally, for the haemolysin III protein, Table 3 in $\mathrm{La}$ 
et al. [30], comparing the nucleotide and amino acid differences of strain D28 and JR11, gives the amino acid differences from other $B$. hyodysenteriae strains compared to WA1 and not the amino acid differences found in D28 as given in Mahu et al. [23].

Since structural studies or deletion mutant studies are not available for those haemolysis associated genes, it is uncertain if the amino acid changes in these two strains indeed alter the functionality of their proteins. However, the unique similarity of the amino acid changes in two, otherwise genetically unrelated strains, that share the same aberrant phenotype and presumably are both low or avirulent, is striking.

The seeder vaccination trial described in this study shows that, although the number of animals that develop SD was not decreased in immunised animals, the speed of spread of SD is significantly reduced in immunised animals compared to sham-immunised animals. Although faecal shedding after challenge was not significantly decreased, pre-immunised animals showed significantly lower faecal scores compared to shamimmunised animals. The absence of a clear IgG response in serum after immunisation is not surprising since it has been demonstrated that after experimental infection, serum antibody levels start to rise after 2-4 weeks and reach their maximum after 4-7 weeks [7]. In this experiment serum samples were taken 17 days post inoculation with the immunizing strain, which might have been too early to see a clear response. Furthermore, since this strain lacks virulence, epithelial damage in the colon can be expected to be absent. Disruption of the colonic mucosa during SD probably permits further penetration of the underlying tissues and blood vessels by bacterial antigen, which could enhance serum antibody response more intense compared to local mucosal stimulation alone [31].

The production of intestinal IgA may play a role in protection against SD. The delay of the onset of SD in immunised pigs correlated with the presence of a substantial local IgA response at the moment of challenge. Earlier, Rees et al. [31, 32] described the presence of colonic IgA and IgA memory cells in gut associated lymphoid tissue in pigs who were re-challenged after recovering from a first or second challenge with $B$. hyodysenteriae strain B204. In those studies the IgA levels in colonic washings or faeces were correlated with a recent exposure, but not with protection against development of clinical SD. This discrepancy with our findings is partially explained by the definition of protection in those earlier studies as the presence or absence of SD, rather than time to development of SD, as used in the current study. Most importantly in those studies, samples were taken only once, at the time of necropsy, which was several weeks after the last exposure to $B$. hyodysenteriae. Indeed in our studies IgA in seeder vaccinated animals rose shortly after the exposure to the challenge strain, but in the seeder vaccinated animals where this rise in IgA upon challenge was absent, the development of SD was not delayed.

For other bacterial enteropathogens like Shigella flexneri, a rise in colonic IgA plays a role in induction of protection by immunization with live attenuated strains [33]. It has been shown that in order to elicit a high and optimal mucosal immune response, multiple doses of the live strain were necessary. Obviously there are differences between the pathogenesis of $S$. flexneri infections and SD, as well as between the responses of their respective hosts. However, both bacteria share the niche of the colon and for both bacteria, serum antibody responses are not linked with protection. It has been demonstrated on several occasions that the number of animals that is protected against SD is significantly higher with increasing numbers of exposures to experimental challenge $[7,12]$ which would be in line with having a higher and prolonged mucosal immune response after multiple exposures.

The link between local IgA and delayed onset of SD opens perspectives for future vaccine development against $B$. hyodysenteriae. Some earlier studies of immunity induced by experimental infection of B. hyodysenteriae point to the importance of mucosal IgA [31, 32]. Recently there has been a developing interest in Th17 cells and interleukin-17A (IL-17) as critical host defence against extracellular pathogens through upregulation of intestinal IgA [34]. In pigs, IL-17 is exclusively produced by $\mathrm{CD} 4^{+}$and $\gamma \delta \mathrm{TCR}^{+} \mathrm{T}$-cells. The importance of CD4+ cells in the immune response following B. hyodysenteriae infection has been described by several authors $[15,35]$. We have demonstrated a ninefold increase in mRNA levels of IL-17A in SD infected pigs before [36]. Taken together, it would be of most interest to determine if CD4+ cells and upregulation of IL-17A are present in the colonic mucosae of pigs immunised with our strain D28 and to explore ways to further optimise the intestinal IgA response. For example, combined parenteral and oral immunisation has been demonstrated to significantly enhance mucosal IgA response for S. flexneri [37] and might be useful in a B. hyodysenteriae immunisation regime as well.

In conclusion, we describe the lack of virulence of the weakly haemolytic B. hyodysenteriae strain D28. Immunisation of pigs by oral inoculation of this strain, significantly slows down the spread of SD compared to sham-immunised animals in a seeder challenge model. This protection was associated with a strong IgA response upon challenge, providing directions for future vaccine development. 


\section{Competing interests}

The authors declare that they have no competing interests.

\section{Authors' contributions}

MM participated in the design of the study, performed the experiments, analysed the data and drafted the manuscript. SC performed statistical analyses and helped to interpret the results. FB participated in the design of the study, helped to interpret the results and edited the manuscript. JZM performed the experiments. FP, AM and FH coordinated the study, participated in the design of the study, helped to interpret the results and edited the manuscript. All authors read and approved the final manuscript.

\section{Acknowledgements}

This work was supported by the Institute for the Promotion of Innovation by Science and Technology in Flanders (IWT Vlaanderen), Brussels, Belgium (Grant IWT Landbouw 100850) and the Industrial Research Fund (IOF) Grant Nr F2014/IOF-ConcepTT/272, and Grant Nr F2015/IOF-Advanced/159. SC is supported by the Research Foundation Flanders (FWO16/PDO/019).

\section{Publisher's Note}

Springer Nature remains neutral with regard to jurisdictional claims in published maps and institutional affiliations.

\section{Received: 24 July 2017 Accepted: 22 August 2017}

Published online: 05 October 2017

\section{References}

1. Alvarez-Ordonez A, Martinez-Lobo FJ, Arguello H, Carvajal A, Rubio P (2013) Swine dysentery: aetiology, pathogenicity, determinants of transmission and the fight against the disease. Int J Environ Res Public Health 10:1927-1947

2. Hidalgo A, Carvajal A, Vester B, Pringle M, Naharro G, Rubio P (2011) Trends towards lower antimicrobial susceptibility and characterization of acquired resistance among clinical isolates of Brachyspira hyodysenteriae in Spain. Antimicrob Agents Chemother 55:3330-3337

3. Mirajkar NS, Davies PR, Gebhart CJ (2016) Antimicrobial susceptibility patterns of Brachyspira species isolated from swine herds in the United States. J Clin Microbiol 54:2109-2119

4. Mahu M, Pasmans F, Vranckx K, De Pauw N, Vande Maele L, Vyt P, Vandersmissen T, Martel A, Haesebrouck F, Boyen F (2017) Presence and mechanisms of acquired antimicrobial resistance in Belgian Brachyspira hyodysenteriae isolates belonging to different clonal complexes. Vet Microbiol 207:125-132

5. Pyorala S, Baptiste KE, Catry B, van Duijkeren E, Greko C, Moreno MA Pomba MC, Rantala M, Ruzauskas M, Sanders P, Threlfall EJ, Torren-Edo J, Torneke K (2014) Macrolides and lincosamides in cattle and pigs: use and development of antimicrobial resistance. Vet J 200:230-239

6. van Duijkeren E, Greko C, Pringle M, Baptiste KE, Catry B, Jukes H, Moreno MA, Pomba MC, Pyorala S, Rantala M, Ruzauskas M, Sanders P, Teale C, Threlfall EJ, Torren-Edo J, Torneke K (2014) Pleuromutilins: use in foodproducing animals in the European Union, development of resistance and impact on human and animal health. J Antimicrob Chemother 69:2022-2031

7. Joens LA, Harris DL, Baum DH (1979) Immunity to swine dysentery in recovered pigs. Am J Vet Res 40:1352-1354

8. Diego R, Lanza I, Carvajal A, Rubio P, Carmenes P (1995) Serpulina hyodysenteriae challenge of fattening pigs vaccinated with an adjuvanted bivalent bacterin against swine dysentery. Vaccine 13:663-667

9. Fernie DS, Ripley PH, Walker PD (1983) Swine dysentery: protection against experimental challenge following single dose parenteral immunisation with inactivated Treponema hyodysenteriae. Res Vet Sci 35:217-221

10. Glock RD, Schwartz KJ, Harris DL (1978) Parenteral immunization of pigs against infection with Treponema hyodysenteriae. Am J Vet Res 39:639-642

11. Hampson DJ, Robertson ID, Mhoma JR (1993) Experiences with a vaccine being developed for the control of swine dysentery. Aust Vet J 70:18-20
12. Olson LD, Dayalu Kl, Schlink GT (1994) Exacerbated onset of dysentery in swine vaccinated with inactivated adjuvanted Serpulina hyodysenteriae. Am J Vet Res 55:67-71

13. Waters WR, Hontecillas R, Sacco RE, Zuckermann FA, Harkins KR, Bassaganya-Riera J, Wannemuehler MJ (2000) Antigen-specific proliferation of porcine CD8aa cells to an extracellular bacterial pathogen. Immunology 101:333-341

14. Waters WR, Pesch BA, Hontecillas R, Sacco RE, Zuckermann FA, Wannemuehler MJ (1999) Cellular immune responses of pigs induced by vaccination with either a whole cell sonicate or pepsin-digested Brachyspira (Serpulina) hyodysenteriae bacterin. Vaccine 18:711-719

15. Waters WR, Sacco RE, Dorn AD, Hontecillas R, Zuckermann FA, Wannemuehler MJ (1999) Systemic and mucosal immune responses of pigs to parenteral immunization with a pepsin-digested Serpulina hyodysenteriae bacterin. Vet Immunol Immunopathol 69:75-87

16. Haesebrouck F, Pasmans F, Chiers K, Maes D, Ducatelle R, Decostere A (2004) Efficacy of vaccines against bacterial diseases in swine: what can we expect? Vet Microbiol 100:255-268

17. Gabe JD, Chang RJ, Slomiany R, Andrews WH, McCaman MT (1995) Isolation of extracytoplasmic proteins from Serpulina hyodysenteriae B204 and molecular cloning of the flaB1 gene encoding a 38-kilodalton flagellar protein. Infect Immun 63:142-148

18. La T, Phillips ND, Reichel MP, Hampson DJ (2004) Protection of pigs from swine dysentery by vaccination with recombinant BmpB, a 29.7 kDa outer-membrane lipoprotein of Brachyspira hyodysenteriae. Vet Microbiol 102:97-109

19. Song Y, La T, Phillips ND, Bellgard MI, Hampson DJ (2009) A reverse vaccinology approach to swine dysentery vaccine development. Vet Microbiol 137:111-119

20. Davis AJ, Smith SC, Moore RJ (2005) The Brachyspira hyodysenteriae ftnA gene: DNA vaccination and real-time PCR quantification of bacteria in a mouse model of disease. Curr Microbiol 50:285-291

21. Holden J, Coloe PJ, Smooker PM (2008) An evaluation of the immunogenicity and protective responses to Brachyspira hyodysenteriae recombinant SmpB vaccination. Vet Microbiol 128:354-363

22. Hyatt DR, ter Huurne AA, van der Zeijst BA, Joens LA (1994) Reduced virulence of Serpulina hyodysenteriae hemolysin-negative mutants in pigs and their potential to protect pigs against challenge with a virulent strain. Infect Immun 62:2244-2248

23. Mahu M, De Pauw N, Vande Maele L, Verlinden M, Boyen F, Ducatelle R, Haesebrouck F, Martel A, Pasmans F (2016) Variation in hemolytic activity of Brachyspira hyodysenteriae strains from pigs. Vet Res 47:66

24. Jenkinson SR, Wingar CR (1981) Selective medium for the isolation of Treponema hyodysenteriae. Vet Rec 109:384-385

25. Verbrugghe E, Vandenbroucke V, Dhaenens M, Shearer N, Goossens J, De Saeger S, Eeckhout M, D'Herde K, Thompson A, Deforce D, Boyen F, Leyman B, Van Parys A, De Backer P, Haesebrouck F, Croubels S, Pasmans F (2012) T-2 toxin induced Salmonella typhimurium intoxication results in decreased Salmonella numbers in the cecum contents of pigs, despite marked effects on Salmonella-host cell interactions. Vet Res 43:22

26. Song Y, Hampson DJ (2009) Development of a multiplex QPCR for detection and quantitation of pathogenic intestinal spirochaetes in the faeces of pigs and chickens. Vet Microbiol 137:129-136

27. Black M, Moolhuijzen P, Barrero R, La T, Phillips N, Hampson D, Herbst W, Barth S, Bellgard M (2015) Analysis of multiple Brachyspira hyodysenteriae genomes confirms that the species is relatively conserved but has potentially important strain variation. PLoS One 10:e0131050

28. Leyman B, Boyen F, Van Parys A, Verbrugghe E, Haesebrouck F, Pasmans F (2011) Salmonella typhimurium LPS mutations for use in vaccines allowing differentiation of infected and vaccinated pigs. Vaccine 29:3679-3685

29. Peters IR, Calvert EL, Hall EJ, Day MJ (2004) Measurement of immunoglobulin concentrations in the feces of healthy dogs. Clin Diagn Lab Immunol $11: 841-848$

30. La T, Rohde J, Phillips ND, Hampson DJ (2016) Comparison of Brachyspira hyodysenteriae isolates recovered from pigs in apparently healthy multiplier herds with isolates from herds with swine dysentery. PLoS One 11:e0160362

31. Rees AS, Lysons RJ, Stokes CR, Bourne FJ (1989) Antibody production by the pig colon during infection with Treponema hyodysenteriae. Res Vet Sci 47:263-269 
32. Rees AS, Lysons RJ, Stokes CR, Bourne FJ (1989) The effect of parenteral immunisation on antibody production in the pig colon. Vet Immunol Immunopathol 23:171-178

33. Keren DF, Kern SE, Bauer DH, Scott PJ, Porter P (1982) Direct demonstration in intestinal secretions of an IgA memory response to orally administered Shigella flexneri antigens. J Immunol 128:475-479

34. Luo Y, Van Nguyen U, de la Rodriguez PYF, Devriendt B, Cox E (2015) F4+ ETEC infection and oral immunization with F4 fimbriae elicits an IL-17-dominated immune response. Vet Res 46:121

35. Hontecillas R, Bassaganya-Riera J, Wilson J, Hutto DL, Wannemuehler MJ (2005) CD4+ T-cell responses and distribution at the colonic mucosa during Brachyspira hyodysenteriae-induced colitis in pigs. Immunology 115:127-135

36. Quintana-Hayashi MP, Navabi N, Mahu M, Venkatakrishnan V, Fernandez HR, Haesebrouck F, Pasmans F, Linden SK (2017) Neutrophil elastase and IL17 expressed in the pig colon during Brachyspira hyodysenteriae infection synergistically with the pathogen induce increased mucus transport speed and production via MAPK3. Infect Immun. doi:10.1128/ IAl.00262-17

37. Keren DF, McDonald RA, Carey JL (1988) Combined parenteral and oral immunization results in an enhanced mucosal immunoglobulin A response to Shigella flexneri. Infect Immun 56:910-915

\section{Submit your next manuscript to BioMed Central and we will help you at every step:}

- We accept pre-submission inquiries

- Our selector tool helps you to find the most relevant journal

- We provide round the clock customer support

- Convenient online submission

- Thorough peer review

- Inclusion in PubMed and all major indexing services

- Maximum visibility for your research

Submit your manuscript at www.biomedcentral com/submit 